\title{
A reinterpretation of extinction in appetitive conditioning
}

\author{
RICHARD D. OLSON, JAMES G. MAY, and GEORGE D. WILLIAMS, JR. \\ University of New Orleans, Lake Front, New Orleans, Louisiana 70122
}

\begin{abstract}
This study compared the effects of response-independent reinforcement and the withdrawal of response-contingent reinforcement on food rewarded barpressiong rates in rats. Neither the resultant decreases in response rate nor subsequent reacquisition performance were differentially affected.It was suggested that the traditional response-contingent reinforcement withdrawal was actually a mild form of punishment and that only the response-independent procedure validly represented extinction.
\end{abstract}

Based on the idea that a habit was eliminated when a response was made ineffective in altering an organism's environment, Davenport and Olson (1968) redefined extinction in the discriminated avoidance paradigm as the presentation of the aversive stimulus independent of $S$ 's response. Using the response-independent presentation of shock as the methodological definition of extinction, Olson, Davenport, and Kamichoff (1971) supported the validity of the procedure by demonstrating the PRE in avoidance conditioning.

A similar conceptualization may also be applied to the elimination of an appetitively conditioned response. Given that a positively reinforcing stimulus has been made response-contingent, the extinction of such a response has traditionally involved the removal of the reinforcing stimulus. Such reinforcement-withdrawal (RW) procedures are usually quite effective, and the response strength quickly returns to baseline level. However, the removal of the reinforcement must definitely be considered a substantial alteration of the organism's environment.

Thus, working by analogy from the avoidance paradigm, extinction of an appetitive response should occur with the presentation of the reinforcing stimulus independent of S's response. In such a situation, the components of the situation are not altered but rather their relationship is changed. Accordingly, response-independent reinforcement (RIR) should be associated with a decrease in response strength that ultimately reaches baseline.

Although carried out to examine other problems, recent studies report data quite relevant to this distinction. Rescorla and Skucy (1969) found that continued food presentation independent of barpressing retarded extinction compared to the conventional RW procedure for S's originally trained on a VI schedule. Comparing the effects of RW and RIR in single schedule and multiple conditions, Boakes (1973) found that under both conditions response rate decreased more rapidly with the former as opposed to the latter condition.

The implication of these studies has been that the retarded response decrement was probably due to superstitious behavior. Working within the framework suggested by Davenport and Olson (1968) and later formalized by Olson and Olson (1971), however, another interpretation might be suggested. Given that the RIR procedure is the proper methodological definition of extinction, the more rapid decrement associated with the no-food procedure might be attributed to punishment. Given asymptotic performance, during acquisition, the total removal of food constitutes the withdrawal of an expected pleasurable stimulus and thus serves as a mild form of punishment. The theoretical work of Mowrer (1960) seems to support such an interpretation.

The purpose of the present study was to continue the investigation of conventional and redefined extinction procedures by examining their effects using a yoked design and to assess the effects of secondary reinforcement in sustaining barpressing.

\section{METHOD}

\section{Subjects}

The Ss were 24 male, Sprague-Dawley rats about 100-days old at the start of the experiment. They were maintained throughout the experiment at $80 \%$ of their normal body weight.

\section{Apparatus}

The experimental chambers were four identical operant boxes (Scientific Prototype Model A-106) connected to appropriate programming modules.

\section{Procedure}

After magazine training, all Ss received six consecutive daily 1-h sessions on a CRF schedule (i.e., each barpress resulted in one 45-mg Noyes pellet). Beginning with the following day, Ss received 13 consecutive daily 1-h sessions on a VR-6 sched ule. The Ss were then divided into four groups, roughly matched for response rate during the last 3 days of the VR training period. The four groups received four different treatments. Group VR contined to be reinforced for barpressing on a VR-6 schedule. Group VR-Y was yoked to Group VR and received a food pellet whenever Group VR received one; this was the response-independent extinction group. Group E received the typical extinction treatment; i.e., all food delivery was stopped. Group E-Y was yoked to Group E and heard the sounds of the empty food dispenser whenever Group E did to assess the efficacy of secondary reinforcement.

These conditions remained operative for six consecutive daily 
1-h sessions (Days 14-20). On the following day, all Ss were again placed on a VR-6 schedule for six consecutive daily 1-h reacquisition sessions (Days 21-26). In all cases the dependent variable was the total number of barpresses made per session.

\section{RESULTS AND DISCUSSION}

The mean number of barpresses per day for each group are presented in Fig. 1, where it is clear that the three experimental groups (VR-Y, E, \& E-Y) exhibited a decreased rate of responding. A mixed analysis of variance performed on the data for the last 13 days of acquisition yielded only the expected days effect $(\mathrm{F}=9.95 ; \mathrm{df}=12,186 ; \mathrm{p}<.01)$, indicating no reliable differences existed between the experimental groups.

A similar analysis performed on the experimental data for Days 14-20 also produced only a days effect $(\mathrm{F}=58.79 ; \mathrm{df}=5,75 ; \mathrm{p}<.01)$, indicating that while the response decrement was very reliable, it was the same for all groups. This point was extremely important as it suggested that both the RW procedure of removing food and redefined RIR procedure of making the food independent of behavior were equally efficacious. Such a result would not be antagonistic to an interpretation that the former procedure was a mild form of punishment while the latter was extinction. Further, the E-Y group performed no better than E or VR-Y, indicating that any secondary reinforcement effects due to magazine noise were either too nugatorical to be observed with the present dependent variable or else simply failed to exist.

The current finding that the response rate returned to baseline level is in conflict with Rescorla and Skucy (1969) and Boakes (1971), both of whom found that the traditional extinction methodology produced a lower response rate asymptote that went below baseline. However, their results are even more suggestive of a punishment interpretation as the more rapid decline and lower asymptote would be expected with a punished rather than an extinguished response.

A final analysis of the reacqusition data (Day 21-26) also yielded only a days effect $(F=20.69$; $d f=11,165$; $\mathrm{p}<.01$ ), which was indicative of the increased bar pressing performance in all groups. Rescorla and Skucy (1969), however, found that the RW group performed reliably more responses during reacquisition. The difference in findings can probably be attributed to either increased length of the extinction period in their study or the differences in the reinforcement schedule used. It is interesting that they found it difficult to explain the rapid rise in responding in the traditional extinction group over a redefined group. Such a finding would, however, again be consistent with the punishment hypotheses. A response suppressed by

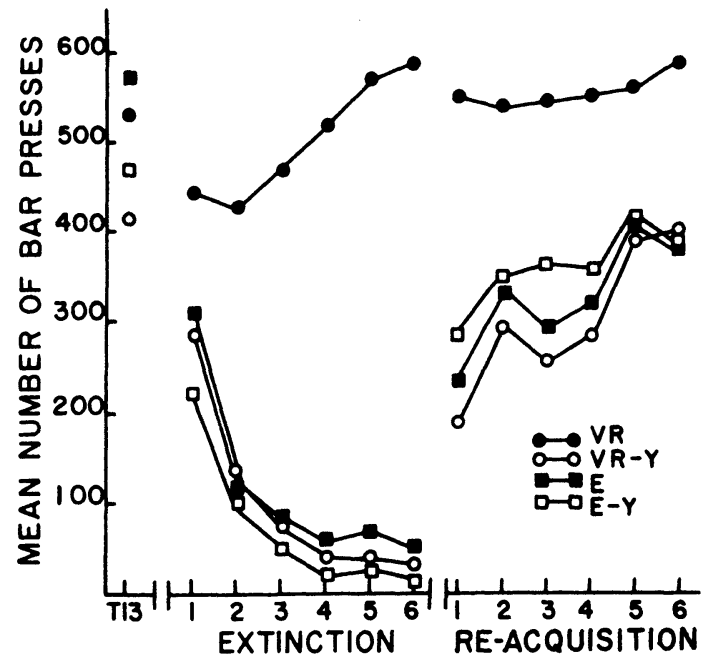

Fig. 1. Mean Number of responses during extinction and acquisition.

continuous punishment has been shown to rise extremely rapidly with the removal of the aversive stimulus (e.g., Azrin \& Holz, 1966). A habit which has been weakened using RIR procedures would be expected to be reacquired more slowly. While additional work is necessary to convincingly show that traditional RW procedures in appetitive conditioning are best considered as a form of punishment, the data in this and earlier studies readily fit such an interpretation even though earlier authors admittedly found them difficult to interpret. Accordingly, it is suggested that only the response-independent procedure can be validly termed extinction and that other forms of habit elimination are, in reality, forms of punishment.

\section{REFERENCES}

Azrin, N. H., \& Holz, W. C. Punishment. In W. K. Honig (Ed.) Operant behavior: Areas of research and application. New York: A ppleton-Century-Crofts, 1966, PP. 380-447.

Boakes, R. A. R esponse decrements produced by extinction and by response-ind ependent reinforcement. Journal of the Experimental A nalysis of Behavior, 1973, 19, 293-302.

Davenport, D. G., \& Olson, R. D. A reinterpretation of extinction in discriminated avoidance. Psychonomic Science, $1968,13,5-6$.

Mowrer, O. H. Learning theory and behavior. New York: Wiley, 1960 .

Olson, R. D., Davenport, D. G., \& Kamichoff, N. C. Discriminated avoidance and the partial reinforcement effect. Psychonomic Science, 1971, 22, 12-14.

Olson, G. A.. \& Olson. R. D. Learning theory. R. D. Olson, R. K. Smith, and G. A. Olson (Eds.) Learning in the classroom: theory and applications. Berkeley: McCutchan. 1971.

Rescorla, R. A., \& Skucy, J. C. Effects o : response-independ ent reinforcers during extinction. Journal of Comparative \& Physiological Psychology, 1969, 67, 381-389.

(Received for publication July 25, 1974.) 